\title{
Novel Applications of Zeiss Libra 200 Cs-Corrected TEM with Energy Filtered Precession Electron Diffraction for Structure Determination of Nanocrystals
}

\author{
S. Nicolopoulos, ${ }^{*}$ D. Bultreys, ${ }^{*}$ G. Benner, ${ }^{* *}$ H. Niebel, ${ }^{* *}$ G. Pavia, ${ }^{* *}$ M. Gemmi*** and B. \\ Janssens $* * * *$ \\ * NanoMEGAS SPRL Blvd Edmond Machtens 79, B-1080 Brussels Belgium \\ ** CarlZeiss NTS, Carl Zeiss Strasse 56, 73447 Oberkochen, Germany \\ *** Dipartimento di Scienze della Terra ' Ardito Desio' Sezione di Mineralogia ,Via \\ Botticelli 23,I-20133 Milano \\ **** NanoMEGAS USA and AppFive LLC AZ 85281 Tempe
}

As the need for research on new nanostructured material increases, reliable characterization of their structure at atomic level is becoming very important. Transmission electron microscopy (TEM) is the ideal technique to characterize nanoparticles through high resolution imaging (HREM) with new generation Zeiss Libra Cs corrected TEM (information limit $<0.08 \mathrm{~nm}$ at $200 \mathrm{KV}$ ).

At the same time, in the last few years there is an increasing trend to use TEM as a tool to solve nanostructures by using uniquely electron diffraction information; Precession Electron Diffraction (PED) is a novel technique [1] that allows solving "ab-initio" crystal nanostructures, by using a collection of PED reflection intensities from different Zone Axis (ZA) under quasi-kinematical conditions (as in X-ray Diffraction). Structure determination "ab-initio" of nanomaterials with PED has been proven to be very successful in TEM for a variety of nanocrystals ranging from oxides, minerals, metals [2] up to pharmaceutical and organics.

A new modular digital precession instrument DigiSTAR [3] is interfaced with the Libra $200 \mathrm{FE}$ and for the first time with an aberration corrected microscope, the Zeiss Libra 200 Cs corrected TEM, allowing both microscopes to perform PED through a dedicated user-friendly modular platform in combination with in-column energy filtering (Fig.1a,b). Precession interface module with Libra 200 Cs TEM allows to work up to $5 \mathrm{deg}$ maximum precession angle and saves in memory different alignments for different PED angles; the module can correct on-line spot size aberration effects caused by high precession angles (Patent pending technique).

The combination of the PED module and the latest generation in-column corrected omega energy filter performance with high acceptance angle (100 $\mathrm{mrad}$ at $\Delta \mathrm{E}=10 \mathrm{eV}$ in Libra 200FE) allows to clearly visualize electron diffraction reflections from the First Order Laue Zone (FOLZ) (Fig. 2 a,b); those reflections appear clearly only by applying energy filtering and are very useful either for space group symmetry determination [2] or to enhance fine structure details for structure determinations using PED. In fact, using Zero Loss (ZL) filtering we found that there is a clear improvement in symmetry related PED [111] intensities as Rsym $=16.7 \%$ in comparison with $\mathrm{Rsym}=26.7 \%$ for non filtered PED [111] pattern (Fig 2c,d). In Fig. 3 a-c we may observe for a mayenite mineral crystal how by increasing precession angle, electron diffraction reflections hugely change intensities from dynamical to quasi-kinematical, showing optimum symmetry information only in combination with filtered PED. Recent work [4] also reveals the improvement of ab-initio structure determination for nanocrystals using in-column ZL and Plasmon Loss filtered PED.

References

[1] R. Vincent and P. Midgley, Ultramicroscopy 53 (1994) 271. 
[2] Ultramicroscopy Special issue New Frontiers in Electron Crystallography vol.107, issue 6-7 June-July 2007 Guest Editors: S.Nicolopoulos, T.Weirich.

[3] NanoMEGAS company: www.nanomegas.com

[4] Kim Jin Gyu et al , Journal of Electron Microscopy to be published

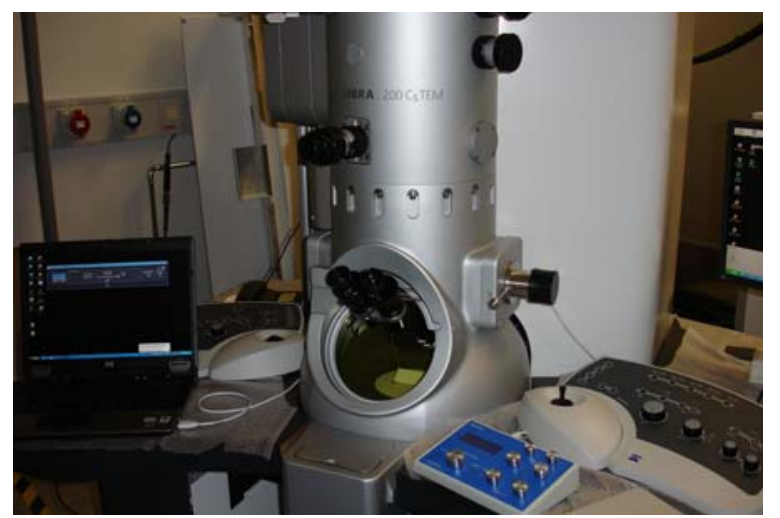

(a)

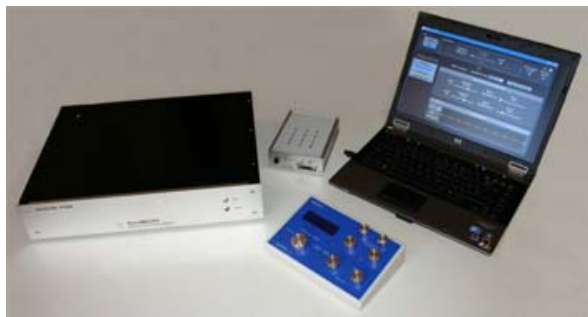

(b)

Fig. 1: (a) Libra 200 Cs corrected microscope equipped with precession DigiSTAR module able to perform ab-initio structure determination of nanocrystals using precession diffraction; (b) DigiSTAR unit with manual interface, main control unit electronics and dedicated PC.

(a)

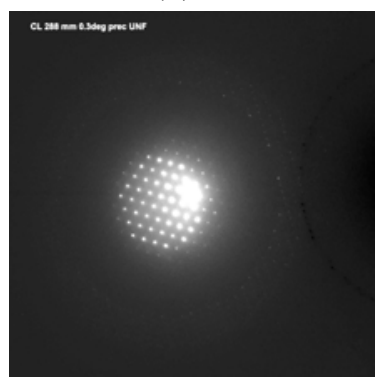

(b)

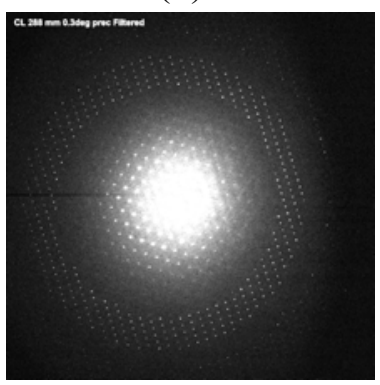

(c)

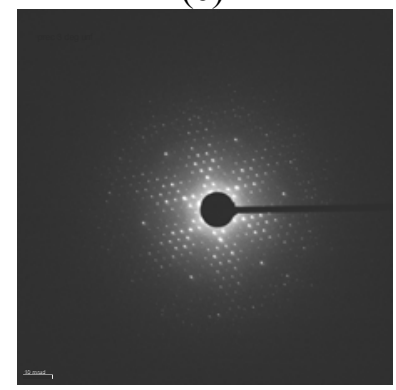

(d)

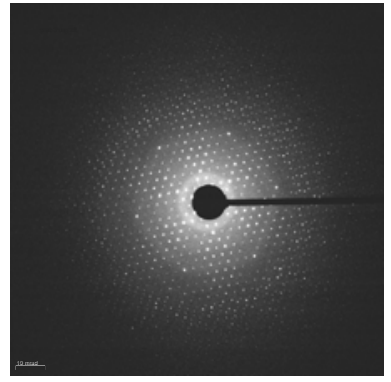

Fig. 2: Mayenite $\mathrm{Ca}_{12} \mathrm{Al}_{14} \mathrm{O}_{33}$ cubic crystal at random orientation (a) non filtered PED pattern (0.3 deg precession angle); (b) same conditions ZL filtered PED: ZOLZ can be now clearly observed; (c) [111] ZA pattern, conventional ED pattern (no precession); (d) [111] precession angle 3 deg, ZL filtered.

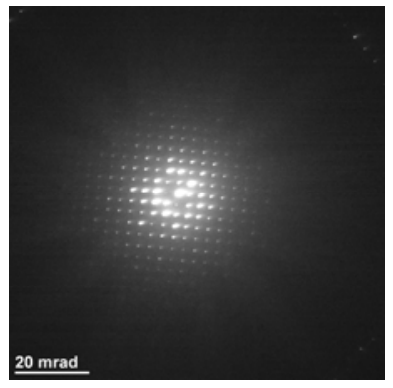

(a)

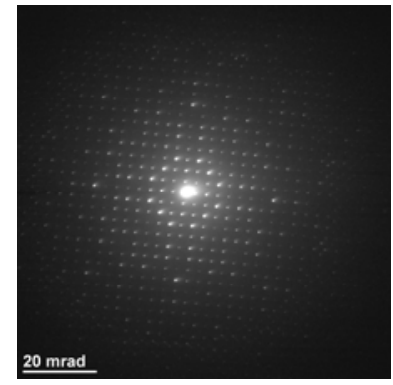

(b)

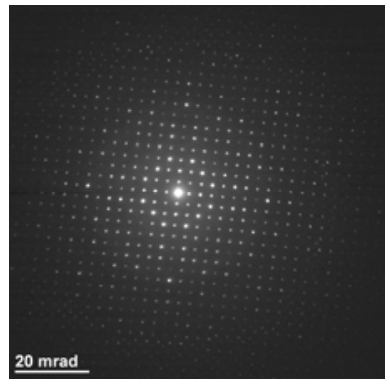

(c)

Fig. 3: (a) [001] ZA mayenite crystal conventional SAED pattern; (b) same ZA crystal 3 deg precession angle unfiltered; (c) same ZA crystal, ZL filtered, 3 deg precession angle. 